\section{INDEX OF NATURAL OBJECTS}

Acanthaster planci Linnaeus, No. 6, 1032

Aconitum neosachalinense, No. 6, 1156

Ampelocissus artemisiifolia Planch., No. 6, 982

Angelica cincta Boissieu (= A amurensis Schischk,

A. anomala auct. non Kitagawa), No. 6, 1136

Angelica viridiflora (Turcz.) Benth. ex Maxim.

( = Gomphopetalum viridiflorum Turcz., Ostericum

viridiflorum (Turcz.) Kitagawa), No. 6, 1136

Apocynum lancifolium, №. 6, 1116

Aquilaria sinensis Gilg, No. 6, 1110

Arum palaestinum Boiss., No. 6, 1075

Aspergillus terreus, No. 6, 1101

Campanula medium, No. 6, 1088

Coelopleurum gmelinii (DC.) Ledeb. (= C. lucidum (L.) Fern.

var. gmelinii (DC.) Hara, Angelica gmelinii (DC.)

M. Pimen.), No. 6, 1136

Crataegus azarolus, No. 6, 1132

Curvularia inaequlis, No. 6, 1120

Euphorbia boissierana, No. 6, 1012

Fusarium sp., No. 6, 1103

Garcinia bracteata, No. 6, 985

Garcinia paucinervis, No. 6, 994

Glycyrrhiza uralensis Fisch. ex DC., No. 6, 1042

Gmelina philippensis Cham., No. 6, 1005

Jacaranda puberula Cham., No. 6, 1143

Junceella sp., No. 6, 1140

Liquidambar formosana Hance, No. 6, 1118

Lophogorgia sp., No. 6, 1140

Lysimachia parvifolia Hemsl., No. 6, 1130

Machilus zuihoensis Hayata var. mushaensis (Lu) Y. C. Liu, No. 6, 978
Malus baccata L., No. 6, 1096

Michelia champaca L., No. 6, 1047

Nelumbo nucifera Gaertn., No. 6, 998

Neptunea arthritica, No. 6, 1099

Nigrospora oryzae (ZJ-2008005), No. 6, 1153

Onopordum acanthium L., No. 6, 1092

Penicillium lividum KMM 4663, No. 6, 1122

Penicillium thomii KMM 4645, No. 6, 1122

Physalis alkekengi L., No. 6, 1094

Pinus silvestris, No. 6, 1009

Piper boehmeriaefolium (Miq.) C. DC. var. tonkinense C. DC., No. 6, 1056

Piriformospora indica, No. 6, 1082

Plantago cornuti Gouan, No. 6, 1001

Plantago lanceolata L., No. 6, 1001

Plantago major L., No. 6, 1001

Plantago media L., No. 6, 1001

Plantago salsa Pall., No. 6, 1001

Plantago stepposa Kupr., No. 6, 1001

Plantago uliginosa F. W. Schmidt, No. 6, 1001

Potentilla discolor Bunge, No. 6, 1128

Prinsepia utilis Royle, No. 6, 1106

Pteris fauriei Hieron., No. 6, 1108

Scutellaria altissima L., No. 6, 1146

Smilax riparia A. DC., No. 6, 989

Taxus canadensis, No. 6, 1050

Tephrosia purpurea (L.) Pers., No. 6, 1113, 1125

Triosteum pinnatifidum Maxim., No. 6, 1149

Typhonium giganteum Engl., No. 6, 1079 